\title{
A BIOMECHANICAL STUDY OF A SUTURE BETWEEN THE DELTOID MUSCLE AND A FREE TENDON GRAFT FOR RECONSTRUCTION OF THE ELBOW EXTENSION
}

\author{
Igor Cizmar ${ }^{\mathrm{a} *}$, Zdenek Florian ${ }^{\mathrm{b}}$, Tomas Navrat ${ }^{\mathrm{b}}$, David Palousek ${ }^{\mathrm{c}}$ \\ ${ }^{a}$ Department of Traumatology, University Hospital Olomouc, Czech Republic \\ ${ }^{b}$ Institute of Solid Mechanics, Mechatronics and Biomechanic, University of Technology, Brno \\ c Institute of Machine and Industrial Design, University of Technology, Brno \\ E-mail: i.cizmar@seznam.cz
}

Received: September 16, 2010; Accepted: November 16, 2010

Key words: Tetraplegic hand/Tendon Transfer/Biomechanics/Spinal cord injury

Aims. It is possible to reconstruct the elbow motion in tetraplegic patients using the posterior portion of the deltoid muscle. In this surgery however, it is a problem to achieve a firm suture between the deltoid muscle and the tendon graft which extends the muscle and is sewn in order to compensate for the plegic musculus triceps brachii function. This study assesses two methods of attachment between muscle and free tendon graft from the biomechanical point of view.

Methods. The assessment was made on 7 fresh-frozen cadaveric samples where the rear portion of the deltoid muscle was sewn with the strip of fascia lata (A1-A7) and 7 samples (B1-B7) where the free tendon graft was attached with a strengthened part of deltoid fascia. The character of the attachment defect was evaluated as strength and elongation parameters using the device Zwick Z020-TND.

Results. The ANOVA showed a statistically significant greater suture solidity connecting the muscle and tendon for group B (B1-B7) than group A. The deformation of the actual suture location was smaller in group B than the deformation of attachment surroundings.

Conclusion. From the biomechanical solidity point of view, it is more efficient to use the strengthened fascia of the deltoid muscle on its inner side for the suture with the tendon graft for reconstruction of the elbow extension in tetraplegic patients.

\section{INTRODUCTION}

The upper limb motion in space, active elbow extension and stabilization of its position are important factors that are absent in the plegia of the musculus triceps brachii in tetraplegic patients with spinal lesions ${ }^{1}$. Reconstruction of the active elbow extension, considered one of the most important surgical procedures in a number of studies on reconstructing the tetraplegic hand, can be done using active muscles, namely musculus biceps brachii or musculus deltoideus ${ }^{2}$. The deltoid muscle is more often used for reconstructions and its suitability from an architectural property viewpoint has been demonstrated experimentally ${ }^{3}$. Moberg first described the transfer method of the deltoid rear portion where this part of the muscle is anchored through a free tendon graft to olecranon ulnae ${ }^{4}$. This reconstruction has been further described in a number of variations mainly in method used and location of tendon graft fixation to the deltoid muscle ${ }^{5-7}$. The problem common to all variations is sufficiently solid technique of tendon graft fixation to loose insertion of the deltoid posterior portion. The deltoid muscle is connected to the humerus at the location of the tuberositas dealtoidei, and the transition is formed by direct attachment of muscle fibres to the periost without tendon transition, i.e. more solid structure suitable for further usage. This fact impedes the possibility of firm suture in this area with a tendon graft that would guarantee the solid attachment of these two structures. Alternatively, use of the deltoid rear portion along with the periost, and thus achieving a "more solid" structure on the transferred muscle is recommended $^{8}$. Hentz has described the transfer with the use of fascia lata as a free tendon graft which in circular fixates the distal part of the muscle using mattress stitches as depicted in (Fig. 1) $)^{0,10}$. Fridén uses the deltoid fascia placed on the inner side $f$ the muscle in the rear portion place as a more solid anchor component, and he sews the tendon graft into this place using continuing cross-stitch technique (Fig. 2) 3, $^{3,1}$. The aim of the present study was to compare the biomechanical properties of both techniques in an experiment on cadaverous material and to assess differences in attachment solidity of the deltoid rear portion and tendon interposit.

\section{MATERIALS AND METHODS}

The experiment was conducted on 15 fresh-frozen cadaveric samples. Fourteen samples fulfilled the structural sample conditions included in the actual biomechanical testing and assessment. The dorsal third of the deltoid muscle was removed from the cadaver along with a bone insertion spina scapulae and part of the acromion. One sample did not possess sufficient bone fragment ensuring 


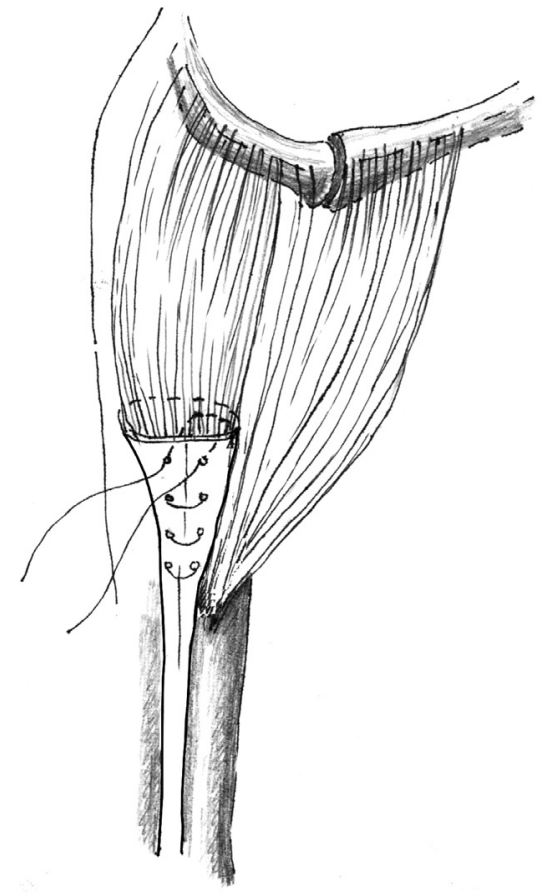

Fig. 1. Schematic representation of Hentz's technique - the transfer of deltoid rear portion with the use of fascia lata as a free tendon graft which in circular fixates distal part of the muscle using mattress stitches.

a

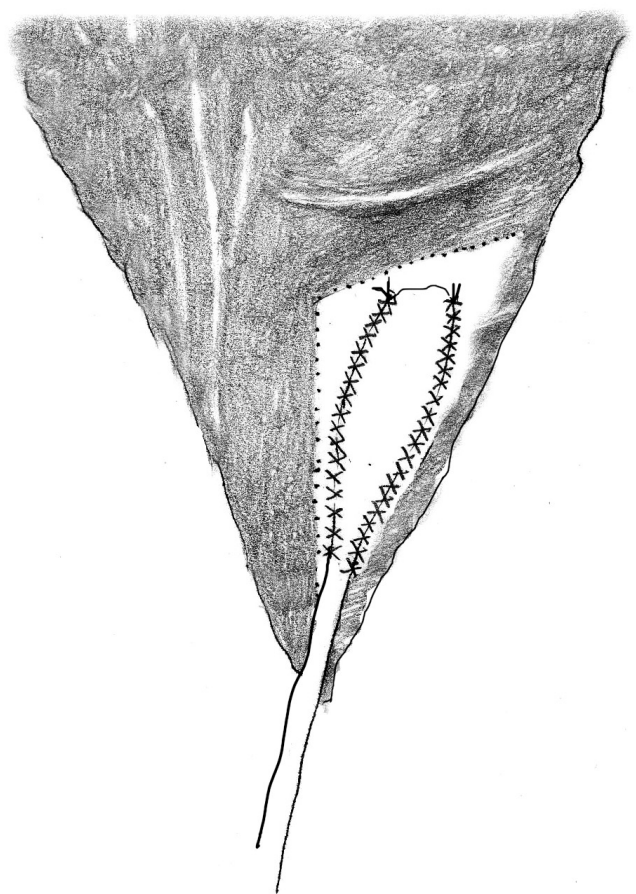

actual fixation to the tear-out machine, and therefore was not assessed. The Hentz technique was used on 7 samples labelled group A (A1-A7), where the insertion of the deltoid muscle with a part of periost tuberisity was cucullately sewn into fascia lata in a length of $20 \mathrm{~cm}$ and width of $4 \mathrm{~cm}$, and fixed with 4 mattress stitches, non-absorbable braided multifilament fibre (PremiCron ${ }^{\circledR}$ 2.0). In a further 7 samples labelled group B(B1-B7), the technique described by Fridén was used, where the tendon tibialis anterior was fixed by its proximal end without muscle fibres using the continuing cross-stitch to the strengthened fascia on the inner deltoid side. Mutual overlapping of the muscle and the tendon was $5 \mathrm{~cm}$ long. Non-absorbable braided multifilament fibre (PremiCron ${ }^{\circledR} 2.0$ ) was used in this variation.

Tolerability assessment of the variations described apropos the attachment of the tendon and muscle tissue took place at the Institute of Solid Mechanics, Mechatronics and Biomechanic, Brno University of Technology using the experimental device Zwick Z 020-TND by Zwick $\mathrm{GmbH} \& \mathrm{Co}$., which is equipped to perform basic biomechanical tests. This is a mechanical, computer-operated machine which enables tests in traction and compression

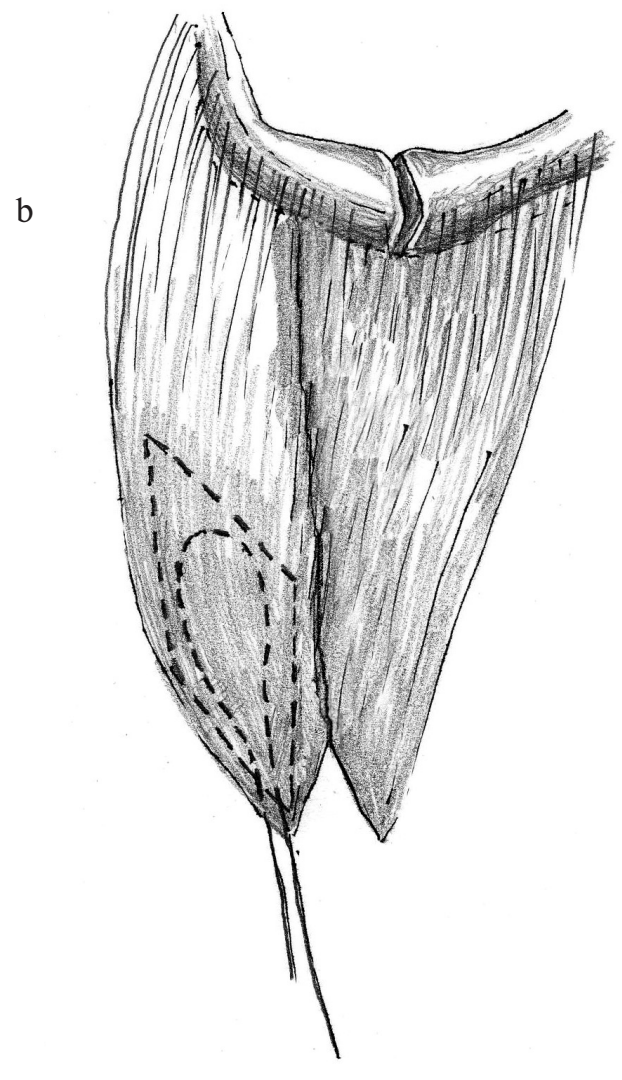

Fig. 2. Variation of deltoid fixation with a tendon graft described by Fridén who has utilized deltoid fascia placed on the inner side of the muscle for more solid attachment using the continuing cross-stitch technique with musculus tibialis anterior tendon.

a) View of the inner side of the deltoid muscle with a marked place where the strengthened fascia is of an inconstant shape.

b) External view of the final attachment tendon/muscle. 
areas during monotonic or cyclic loading. For imaging of small shifts it is equipped with a sensor of elongation Multi-sens to an accuracy of $0.1 \mu \mathrm{m}$. The experiment focused on assessment of defect, and mainly force intensity which caused the defect, for the described attachment techniques of muscle and tendon. Taking into consideration the mechanical properties of muscle, the loading proceeded at the low speed of $0.5 \mathrm{~mm} / \mathrm{s}$. Anchoring the experimental samples into the tear-out machine is described in (Fig. 3).

The measured maximal force values were divided into 2 according to method of suture (A1-A7, B1-B7). ANOVA

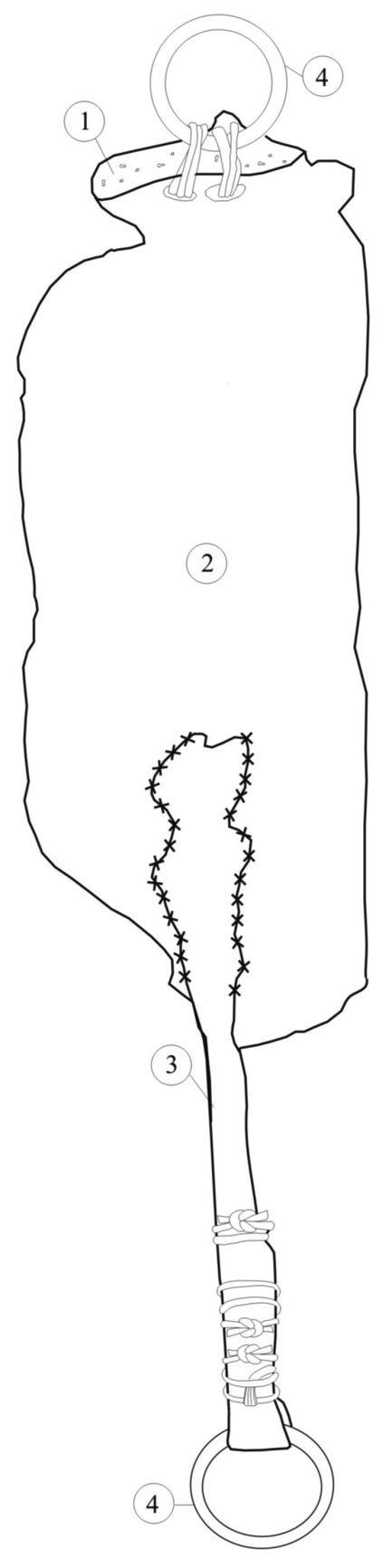

Fig. 3. Anchoring the experimental samples into the tearout machine: 1 - bone, 2 - muscle, 3 - tendon, 4 - ring for fixation. was used to test whether the differences between groups A and B were statistically significant, and hence whether the method of suture had an effect on degree of maximal force necessary to disrupt the sample. The results were also analysed using the non parametric test, Kruskal Wallis.

In addition to the assessment of the maximal force necessary for sample disruption, elongation assessment was carried out from the muscle-tendon attachment technique point of view. Th experiment was recorded with a video camera in high definition. Selected sample reference points were monitored in elongation assessment, namely in area of structure attachment, in the muscle tissue itself and in the tendon. Mutual position and length along the reference points were determined. These quantities were evaluated in gradually increasing loading, in progressing values: $0 ; 1 ; 5 ; 10 \mathrm{~N}$.

\section{RESULTS}

The results are shown in Table 1. Graphic representation is depicted in the box chart (Fig. 4) with statistics.

On the basis of the analysis of variance (ANOVA), at the significance level of $5 \%$ we rejected the hypothesis that population mean values were the same $(p=0.046)$. This

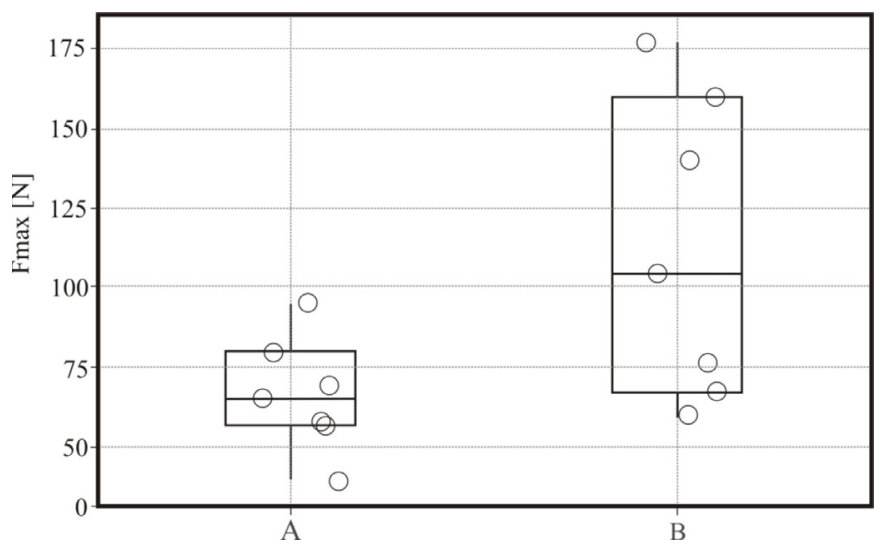

Fig. 4. Box chart, the points represent particular realizations in tested groups.

Table 1. Descriptive statistical assessment of measured samples from both groups.

\begin{tabular}{|l|c|c|}
\hline \multicolumn{1}{|c|}{ Fmax $[\mathrm{N}]$} & A & B \\
\hline Number of samples & 7 & 7 \\
\hline Minimal value & 39.0 & 60.0 \\
\hline Average value & 65.9 & 112.0 \\
\hline Median & 65.0 & 104.0 \\
\hline Standard deviation & 17.9 & 47.3 \\
\hline Maximal value & 95.0 & 177.0 \\
\hline
\end{tabular}




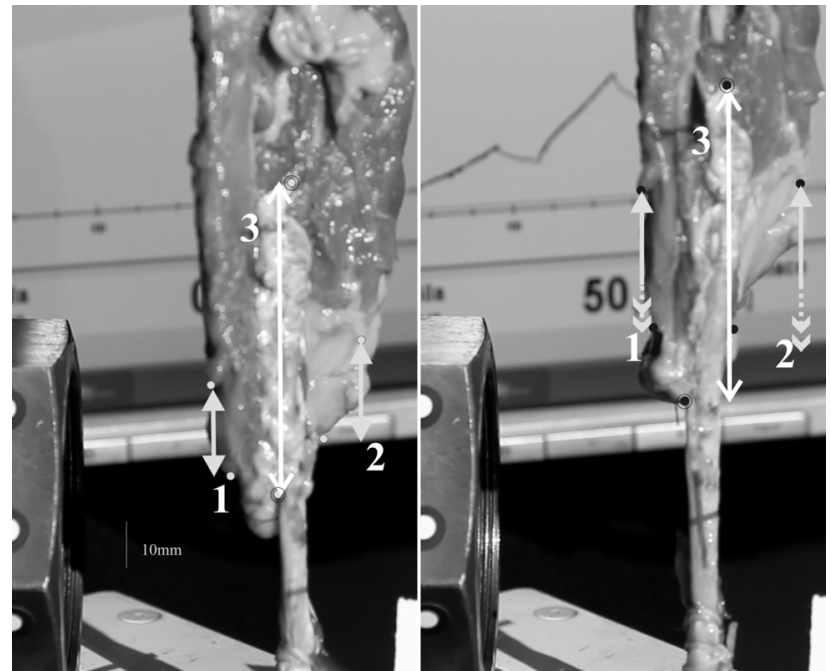

Fig. 5. The deformation of the actual attachment in samples of group B(B1-B7) after loading has been significantly smaller (line 3 ) than the deformation of attachment surroundings (line 1,2).

implies that statistically significant difference in attachment solidity for different methods of suture was proven. The same conclusion was drawn from the non-parametric test (Kruskal-Wallis) $(\mathrm{p}=0.048)$.

In the case of the elongation assessment for the two groups divided by technique of muscle attachment, significant differences were found for deformation and transformation of areas. In the case of the technique A significant deformations in the attachment area were found at low loading values, whereas in the area of muscle tissue the deformations were small. In the case of the technique B concerning the attachment of the tendon and muscle tissue the attachment length changed insignificantly throughout the whole experiment. The deformation of the muscle tissue itself, or possibly tendon, was significantly greater. The length of relatively solid attachment in case B did not influence the elongation of the sample (muscle) in a negative way because the load in configuration $\mathrm{B}$ was well distributed through the whole muscle volume (Fig. 5).

\section{DISCUSSION}

The results show better biomechanical attachment properties where the strengthened fascia of the deltoid muscle was used. However there was wide variability given the relatively homogenous group of samples where the fascia lata was fixed to the muscle fascicle using mattress stitches.It may be assumed that the results are compromised by posthumous muscle atonia. However, significant structural changes are excluded given the early posthumous application. The variability of maximal force in group B was probably also caused by the anatomical variability of the strengthened fascia, its size and placement (Fig. 2a). We cannot predict the influence of non-viable musculature on structural solidity but it is obvious that the attachment solidity was considerably bigger in group B than the place of suture in group A. Similar results for samples of group B are also applicable in the case of deformation of the actual attachment before its disruption. The testing did not quantify in detail the significant influence of a large number of variables on the deformation properties of the muscle - size, material, number of stitches etc.

The observed differences in suture solidity and the extent of its elongation after loading, concerning the type of the technique used, participate in post-operative elongation of the transferred muscle with function loss ${ }^{12}$. Experimental values are altered in clinical application, and by different interpretations of described suture techniques by particular surgeons.

\section{CONCLUSION}

The AVOVA showed a statistically significant greater solidity of suture connecting the muscle and tendon for samples of group B(B1-B7). The deformation during elongation in samples of group A(A1-A7) was continuous up to full sample disruption, and the smaller deformation of actual attachment towards its surroundings was not observed, for group A as it was for group B.

\section{ACKNOWLEDGEMENTS}

This study has been supported by grant IGA MZ: NS9620-3/2008

\section{REFERENCES}

1. Čižmář I, Zálesák B, Pilny J, Dráč P, Fialová J. Possible restorations of the upper extremity motion in tetraplegic patients - 5 year clinical experience. Biomed Pap Med Fac Univ Palacky Olomouc Czech Repub 2006;150(2):313-9.

2. Fridén J, Reinholdt C. Current concepts in reconstruction of hand function in tetraplegia. Scand J Surg 2008;97:341-6.

3. Fridén J, Lieber RL. Quantitative evaluation of the posterior deltoid to triceps tendon transfer based on muscle architectural properties. J Hand Surg 2001;26A(1):147-55.

4. Moberg E. Surgical treatment for absent single-hand grip and elbow extension in quadriplegia. J Bone Joint Surg 1975; 57A(2):196-206.

5. Bryan R. The Moberg deltoid-triceps replacement and keypinch operations in quadriplegia: preliminary experience. Hand 1977;9(3):207-14.

6. Ejeskar A, Dallhof A. Results of reconstructive surgery in the upper limb of tetraplegic patients. Paraplegia 1988;26:204-8.

7. Ejeskar A. Upper limb surgical rehabilitation in high-level tetraplegia. Hand Clin 1988;4:585-99.

8. Falconer D. Tendon transfers about the shoulder and elbow in the spinal cord injured patient. Hand Clin 1988;4:211-21.

9. Hentz V, Brown M, Keoshian L. Upper limb reconstruction in quadriplegia: functional assessment and proposed treatment modifications. J Hand Surg 1983;8(2):119-31.

10. Hentz VR, Leclercq C. Surgical rehabilitation of active elbow extension In: Hentz VR, Leclercq C, editors. Surgical rehabilitation 
of the elbow extension

of the upper limb in tetraplegia London: W.B.Saunders; 2002. p.97-117.

11. Fridén J. Reconstruction of elbow in tetraplegia In: Fridén J, editor. Tendon transfers in reconstructive hand surgery London: Taylor\&Francis; 2005. p.91-102.

12. Fridén J, Ejeskãr A, Dahlgren A, Lieber RL. Protection of the deltoid-to-triceps tendon transfer repair sites. J Hand Surg 2000;25A:144-9. 
